$\underline{\xi}=$ 园

\title{
Comparative study of the dynamics of Mimas-Tethys system at different resonances
}

\author{
Sarita Jha $^{1 *}$, Govind Kumar Jha ${ }^{2}$ \\ ${ }^{I}$ Department of Mathematics, K. B. Women's College, Hazaribag, Jharkhand Mb. 8051140448 \\ ${ }^{2}$ Department of Mathematics, Vinoba Bhave University, Hazaribag, Jharkhand Mb.09798315444 \\ *Corresponding author E-mail: saritajhakbw.vbu@gmail.com
}

\begin{abstract}
Here we have done the comparative study on the dynamics of Mimas-Tethys system at $\mathrm{i}_{1} \mathrm{i}_{3}, \mathrm{i}_{1} \mathrm{i}_{3} \mathrm{e}_{3}, \mathrm{i}_{1}^{2} \mathrm{e}_{3}, \mathrm{i}_{3}{ }^{2} \mathrm{e}_{3}$ and $\mathrm{i}_{3}{ }^{2}, \mathrm{i}_{1} \mathrm{i}_{3} \mathrm{e}_{3}, \mathrm{i}_{1}^{2} \mathrm{e}_{3}, \mathrm{i}_{3}{ }^{2} \mathrm{e}_{3}$ resonances along with secular resonance of all inner satellites and Saturn's oblateness. We have drawn Poincare surface of sections and Time-series graph to compare their effect.
\end{abstract}

Keywords: Secular Resonance; Three-Body Problem; Disturbing Function; Oblateness and Poincare Surface of Section.

\section{Introduction}

Allan [1] and Sinclair [2] investigated the dynamics of the MimasTethys system under the hypothesis of circular orbits. Allan found (backward in time) the values of the satellites inclinations before capture in the present resonance to be $\mathrm{i}_{1}=0.42^{\mathrm{O}}$ and $\mathrm{i}_{3}=1.05^{\mathrm{O}}$ Sinclair found the probability of capture is 0.04 .

Champenois and Vienne [3],[4] numerically investigated the role of 200 year period and found Mimas's inclination before capture may have been higher (up to 0.7 degree) or lower (down to 0.03 degree) than the previously considered 0.42 degree. Furthermore, Tethys's eccentricity on capture may have been quite higher $(0.0008$ versus 0$)$. This value of eccentricity was found by a method which takes chaos into account. They also found that probability of capture in $i_{1} i_{3}$ resonance is 0.76 at eccentricity of Tethys is 0.0008 .

Jha and Agrawal [5] have done the comparative study of the dynamics of Mimas-Tethys at $\mathrm{i}_{1}{ }^{2}, \mathrm{i}_{1} \mathrm{i}_{3}$ and $\mathrm{i}_{3}{ }^{2}$ resonances along with three third-order resonances without considering the secular term of all inner satellites and oblateness of Saturn. Jha and Agrawal [6] have studied the effect of secular resonance of Enceladus on Mimas-Tethys system. Jha and Jha [7-9] have studied the secular resonance effect of Dione, Rhea and Titan on this system.

Here we are extending the work of Jha and Agrawal [6] by considering the effect of secular term of all inner satellites along with Saturn's oblateness.

The physical model was taken to be Mimas and Tethys on eccentric orbits inclined on the equatorial plane of Saturn. Saturn's gravitational momenta are essential as they provide the main contribution to the orbital precession rates and we had to take into account the lowest degree oblateness terms $\mathrm{J}_{2}, \mathrm{~J}_{4}, \mathrm{~J}_{2}{ }^{2}, \mathrm{~J}_{6}, \mathrm{~J}_{2} \mathrm{~J}_{4}$ and $\mathrm{J}_{2}{ }^{3}$ (See table 2 ) also the actions of the Japet in the equations whereas the Sun and the small satellites of
Saturn are not taken into account because of their weak effects in the generations of the purturbative frequency $\sigma$.

Here the notations $\mathrm{a}_{1}, \mathrm{n}_{1}, \mathrm{e}_{1}, \mathrm{i}_{1}, \gamma_{1}, \varpi_{1}, \Omega_{1}$ and $\lambda_{1}$ are orbital semimajor axis, mean motion, eccentricity, inclination, sine of semi inclination, longitudes of periapse, longitude of ascending node and mean longitude of Mimas respectively. Corresponding notions with subscript 2, 3, 4, 5 and 6 refer to the Enceladus, Tethys, Dione, Rhea and Titan's orbital elements. Small $\mathrm{m}_{1}, \mathrm{~m}_{2}, \mathrm{~m}_{3}, \mathrm{~m}_{4}$, $\mathrm{m}_{5}$ and $\mathrm{m}_{6}$ stand for Mimas, Enceladus, Tethys, Dione, Rhea and Titan's masses with unit of Saturn.

\section{Equation of motion when the system is} locked in ${ }^{2}{ }^{2}, \mathrm{i}_{1}{ }^{2} \mathrm{e}_{3}, \mathrm{i}_{1} \mathrm{i}_{3} \mathrm{e}_{3}$ and $\mathrm{i}_{3}{ }^{2} \mathrm{e}_{3}$ resonances

(Equation of motion is taken from Jha and Agrawal [5].

$$
\begin{aligned}
& \ddot{\chi}=\mathrm{A}_{\chi} \operatorname{Sin}[\chi]+\mathrm{A}_{\psi+\varphi} \operatorname{Sin}[\psi+\varphi]+\mathrm{A}_{\psi} \operatorname{Sin}[\psi]+\mathrm{A}_{\psi-\varphi} \operatorname{Sin}[\psi-\varphi] \\
& =\mathrm{A}_{\chi}\left[\operatorname{Sin}(\chi)+\mathrm{A}_{\chi 1} \operatorname{Sin}\left(\frac{3}{2} \chi+\frac{3}{2}\left(\Omega_{1}-\Omega_{3}\right)+\sigma\right)\right. \\
& +\mathrm{A}_{\chi 2} \operatorname{Sin}\left(\frac{1}{2} \chi+\frac{1}{2}\left(\Omega_{1}-\Omega_{3}\right)+\sigma\right) \\
& +\mathrm{A}_{\chi^{3}} \operatorname{Sin}\left[-\frac{1}{2} \chi-\frac{1}{2}\left(\Omega_{1}-\Omega_{3}\right)+\sigma\right] \\
& +\left(\frac{\mathrm{d}^{2} \varphi}{\mathrm{dt}^{2}}\right)_{\mathrm{O}}+\left(\frac{\mathrm{d}^{2} \sigma}{\mathrm{dt}^{2}}\right)_{\mathrm{o}}+\left(\frac{\mathrm{d}^{2} \varphi}{\mathrm{dt}^{2}}\right)_{\mathrm{s}}+\left(\frac{\mathrm{d}^{2} \sigma}{\mathrm{dt}^{2}}\right)_{\mathrm{S}}
\end{aligned}
$$


Where,

$$
\begin{aligned}
& \chi=2 \lambda_{1}-4 \lambda_{3}+2 \Omega_{3}, \\
& \begin{aligned}
\psi+\varphi & =3 \lambda_{1}-6 \lambda_{3}+2 \Omega_{1}+\varpi_{3} \\
& =\frac{3}{2}\left(\chi+\Omega_{1}-\Omega_{3}\right)+\sigma
\end{aligned} \\
& \begin{aligned}
\psi & =\lambda_{1}-2 \lambda_{3}+\Omega_{1}-\Omega_{3}+\varpi_{3} \\
& =\frac{1}{2}\left(\chi+\Omega_{1}-\Omega_{3}\right)+\sigma
\end{aligned}
\end{aligned}
$$

And

$$
\begin{aligned}
\psi-\varphi & =-\lambda_{1}+2 \lambda_{3}-2 \Omega_{3}+\varpi_{3} \\
& =-\frac{1}{2}\left(\chi+\Omega_{1}-\Omega_{3}\right)+\sigma
\end{aligned}
$$

With

$\sigma=\mathrm{ft}+\sigma_{0}$,

$\mathrm{A}_{\chi}=\left(6 \mathrm{n}_{1}^{2} \mathrm{~m}_{3} \alpha_{13}+24 \mathrm{n}_{3}^{2} \mathrm{~m}_{1}\right)\left(-\mathrm{f}_{0}\left(\alpha_{13}\right)\right) \gamma_{3}^{2}$

$A_{\chi 1}=-3 e_{3} \frac{f_{1}\left(\alpha_{13}\right)}{f_{0}\left(\alpha_{13}\right)} \frac{\gamma_{1}^{2}}{\gamma_{3}^{2}}, A_{\chi 2}=-e_{3} \frac{f_{2}\left(\alpha_{13}\right)}{f_{0}\left(\alpha_{13}\right)} \frac{\gamma_{1}}{\gamma_{3}}, A_{\chi 3}=e_{3} \frac{f_{3}\left(\alpha_{13}\right)}{f_{0}\left(\alpha_{13}\right)}$

The value of $f_{0}\left(\alpha_{13}\right), f_{1}\left(\alpha_{13}\right), f_{2}\left(\alpha_{13}\right)$ and $f_{3}\left(\alpha_{13}\right)$ depend on the Laplace's Coefficients $b_{\mathrm{s}}^{(\mathrm{k})}\left(\alpha_{\mathrm{ij}}\right)$ and given in table 1 .

\section{Equation of motion when the system is}

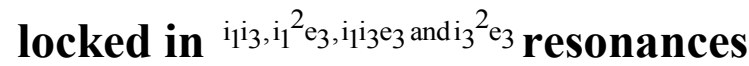

$$
\begin{aligned}
& \frac{\mathrm{d}^{2} \varphi}{\mathrm{dt}^{2}}=\mathrm{A}_{0}\left(\begin{array}{l}
\operatorname{Sin} \varphi+\mathrm{A}_{1} \operatorname{Sin}\left(\frac{3}{2} \varphi+\mathrm{ft}+\sigma_{0}\right) \\
+\mathrm{A}_{2} \operatorname{Sin}\left(\frac{1}{2} \varphi+\mathrm{ft}+\sigma_{0}\right) \\
+\mathrm{A}_{3} \operatorname{Sin}\left(-\frac{1}{2} \varphi+\mathrm{ft}+\sigma_{0}\right)
\end{array}\right)+ \\
& \left(\frac{\mathrm{d}^{2} \varphi}{\mathrm{dt}^{2}}\right)_{\mathrm{O}}+\left(\frac{\mathrm{d}^{2} \sigma}{\mathrm{dt}^{2}}\right)_{\mathrm{O}} \\
& +\left(\frac{\mathrm{d}^{2} \varphi}{\mathrm{dt}^{2}}\right)_{\mathrm{S}}+\left(\frac{\mathrm{d}^{2} \sigma}{\mathrm{dt}^{2}}\right)_{\mathrm{S}}
\end{aligned}
$$

Where

$$
\begin{aligned}
& \varphi=2 \lambda_{1}-4 \lambda_{3}+\Omega_{1}+\Omega_{3}, \\
& \begin{aligned}
\psi+\varphi & =3 \lambda_{1}-6 \lambda_{3}+2 \Omega_{1}+\varpi_{3} \\
& =\frac{3}{2} \varphi+\sigma
\end{aligned} \\
& \psi=\lambda_{1}-2 \lambda_{3}+\Omega_{1}-\Omega_{3}+\varpi_{3} \\
& =\frac{1}{2} \varphi+\sigma
\end{aligned}
$$

$$
\begin{aligned}
\psi-\varphi & =-\lambda_{1}+2 \lambda_{3}-2 \Omega_{3}+\varpi_{3} \\
& =-\frac{1}{2} \varphi+\sigma
\end{aligned}
$$

With

$$
\sigma=\mathrm{ft}+\sigma_{0} \text { where } \sigma_{0}=-0.031391995
$$

And

$\mathrm{A}_{0}=\left(12 \mathrm{n}_{1}^{2} \mathrm{~m}_{3} \alpha_{13}+48 \mathrm{n}_{3}^{2} \mathrm{~m}_{1}\right) \mathrm{f}_{0}\left(\alpha_{13}\right) \gamma_{1} \gamma_{3}$

$A_{1}=\frac{3}{2} e_{3} \frac{f_{1}\left(\alpha_{13}\right)}{f_{0}\left(\alpha_{13}\right)} \frac{\gamma_{1}}{\gamma_{3}}, A_{2}=\frac{1}{2} e_{3} \frac{f_{2}\left(\alpha_{13}\right)}{f_{0}\left(\alpha_{13}\right)}$

and $A_{3}=-\frac{1}{2} \frac{f_{3}\left(\alpha_{13}\right)}{f_{0}\left(\alpha_{13}\right)} \frac{\gamma_{3}}{\gamma_{1}}$

The value of $f_{0}\left(\alpha_{13}\right), f_{1}\left(\alpha_{13}\right), f_{2}\left(\alpha_{13}\right)$ and $f_{3}\left(\alpha_{13}\right)$ depend on the Laplace's Coefficients $b_{\mathrm{s}}^{(\mathrm{k})}\left(\alpha_{\mathrm{ij}}\right)$ and given in table 1 .

With

$$
\begin{aligned}
& \left(\frac{\mathrm{d}^{2} \lambda_{1}}{\mathrm{dt}^{2}}\right)_{\mathrm{S}}=-2\left[\sum_{\mathrm{i}=2}^{5} \mathrm{~m}_{\mathrm{i}} \alpha_{1 \mathrm{i}}^{2} \frac{\mathrm{d}^{2} \mathrm{~A}\left(\alpha_{1 \mathrm{i}}\right)}{\mathrm{d} \alpha_{1 \mathrm{i}}^{2}}\right] \frac{\mathrm{dn}_{1}}{\mathrm{dt}} \\
& \left(\frac{\mathrm{d}^{2} \Omega_{1}}{\mathrm{dt}^{2}}\right)_{\mathrm{S}}=\frac{1}{2}\left[\sum_{\mathrm{i}=2}^{5} \mathrm{~m}_{\mathrm{i}} \alpha_{1 \mathrm{i}} \mathrm{C}\left(\alpha_{1 \mathrm{i}}\right)\right] \frac{\mathrm{dn}_{1}}{\mathrm{dt}}
\end{aligned}
$$

$$
\left(\frac{\mathrm{d}^{2} \Omega_{3}}{\mathrm{dt}^{2}}\right)_{\mathrm{S}}=\frac{1}{2}\left[\sum_{\mathrm{i}=1}^{2} \mathrm{~m}_{\mathrm{i}} \mathrm{C}\left(\alpha_{\mathrm{i} 3}\right)+\sum_{\mathrm{i}=4}^{6} \mathrm{~m}_{\mathrm{i}} \alpha_{3 \mathrm{i}} \mathrm{C}\left(\alpha_{3 \mathrm{i}}\right)\right] \frac{\mathrm{dn}_{3}}{\mathrm{dt}}
$$

$\left(\frac{\mathrm{d}^{2} \varpi_{3}}{\mathrm{dt}^{2}}\right)_{\mathrm{S}}=2\left[\sum_{\mathrm{i}=1}^{2} \mathrm{~m}_{\mathrm{i}} \mathrm{B}\left(\alpha_{\mathrm{i} 3}\right)+\sum_{\mathrm{i}=4}^{6} \mathrm{~m}_{\mathrm{i}} \alpha_{3 i} \mathrm{~B}\left(\alpha_{3 i}\right)\right] \frac{\mathrm{dn}_{3}}{\mathrm{dt}}$

$$
\left(\frac{\mathrm{d}^{2} \lambda_{3}}{\mathrm{dt}^{2}}\right)_{\mathrm{S}}=2\left[\begin{array}{c}
\sum_{\mathrm{i}=1}^{2}{ }^{-m_{\mathrm{i}}}\left\{\alpha_{\mathrm{i} 3} \frac{\mathrm{dA}\left(\alpha_{\mathrm{i} 3}\right)}{\mathrm{d} \alpha_{\mathrm{i} 3}}+\mathrm{A}\left(\alpha_{\mathrm{i} 3}\right)\right. \\
+\sum_{\mathrm{i}=4}^{6} \mathrm{~m}_{\mathrm{i}} \alpha_{3 i}{ }^{2} \frac{\mathrm{dA}\left(\alpha_{3 i}\right)}{\mathrm{d} \alpha_{3 i}}
\end{array}\right] \frac{\mathrm{dn}_{3}}{\mathrm{dt}}
$$

(We are not considering any changes in semi major axis of any satellites)

And

$$
\begin{aligned}
& \left(\frac{\mathrm{d}^{2} \varphi}{\mathrm{dt}^{2}}\right)_{\mathrm{S}}=\left(\frac{\mathrm{d}^{2} \lambda_{1}}{\mathrm{dt}^{2}}\right)_{\mathrm{S}}-2\left(\frac{\mathrm{d}^{2} \lambda_{3}}{\mathrm{dt}^{2}}\right)_{\mathrm{S}}+\frac{1}{2}\left[\left(\frac{\mathrm{d}^{2} \Omega_{1}}{\mathrm{dt}^{2}}\right)_{\mathrm{S}}+\left(\frac{\mathrm{d}^{2} \Omega_{3}}{\mathrm{dt}^{2}}\right)_{\mathrm{S}}\right] \\
& \left(\frac{\mathrm{d}^{2} \sigma}{\mathrm{dt}^{2}}\right)_{\mathrm{S}}=\frac{1}{2}\left(\frac{\mathrm{d}^{2} \Omega_{1}}{\mathrm{dt}^{2}}\right)_{\mathrm{S}}-\frac{3}{2}\left(\frac{\mathrm{d}^{2} \Omega_{3}}{\mathrm{dt}^{2}}\right)_{\mathrm{S}}+\left(\frac{\mathrm{d}^{2} \varpi_{3}}{\mathrm{dt}^{2}}\right)_{\mathrm{S}}
\end{aligned}
$$

Now we will find the terms due to Oblateness of Saturn. Saturn's gravitational momenta are quite important so that we have, in order to get the full variations of the mean longitudes, nodes and pericentres due to the oblateness, taken into account the lowest degree terms with $\mathrm{J}_{2}, \mathrm{~J}_{4}, \mathrm{~J}_{2}{ }^{2}, \mathrm{~J}_{6}, \mathrm{~J}_{2} \mathrm{~J}_{4}$ and $\mathrm{J}_{2}{ }^{3}$ (See Table 2) as a factor (see [10]). Values of $\alpha_{i j}, A\left(\alpha_{i j}\right), B\left(\alpha_{i j}\right), C\left(\alpha_{i j}\right), \frac{d A\left(\alpha_{i j}\right)}{d \alpha_{i j}}$ for Every Pair $(i, j),(i<j)$ Involving Mimas, Enceladus, Tethys, Dione, Rhea and Titan are given in Table 3. The other terms are taken constant. We then get ( $a_{e}$ is the equatorial radius of Saturn). 


$$
\begin{aligned}
& \left(\frac{\mathrm{d}^{2} \Omega_{3}}{\mathrm{dt}^{2}}\right)_{\mathrm{o}}=\left[\begin{array}{l}
\frac{-3}{2} \mathrm{~J}_{2}\left(\frac{\mathrm{a}_{\mathrm{e}}}{\mathrm{a}_{3}}\right)^{2}\left(1+2 \mathrm{e}_{3}^{2}-2 \gamma_{3}^{2}\right) \\
+\mathrm{a}_{3}^{-4}\left(\frac{15}{4} \mathrm{~J}_{4}-\frac{45}{8} \mathrm{~J}_{2}^{2}\right)
\end{array}\right] \frac{\mathrm{dn}_{3}}{\mathrm{dt}} \\
& -6 J_{2} n_{3}\left(\frac{a_{e}}{a_{3}}\right)^{2}\left(e_{3} \frac{d e_{3}}{d t}-\gamma_{3} \frac{d \gamma_{3}}{d t}\right) \\
& \left(\frac{\mathrm{d}^{2} \lambda_{1}}{\mathrm{dt}^{2}}\right)_{\mathrm{O}}=\left[\begin{array}{l}
3 \mathrm{~J}_{2}\left(\frac{\mathrm{a}_{\mathrm{e}}}{\mathrm{a}_{1}}\right)^{2}\left(1+\frac{7}{4} \mathrm{e}_{1}^{2}-7 \gamma_{1}^{2}\right) \\
+\mathrm{a}_{1}^{-4}\left(-\frac{15}{4} \mathrm{~J}_{4}+\frac{160}{11} \mathrm{~J}_{2}^{2}\right)
\end{array}\right] \frac{\mathrm{dn}_{1}}{\mathrm{dt}} \\
& -42 \mathrm{~J}_{2} \mathrm{n}_{1} \gamma_{1}\left(\frac{\mathrm{a}_{\mathrm{e}}}{\mathrm{a}_{1}}\right)^{2} \frac{\mathrm{d} \gamma_{1}}{\mathrm{dt}}
\end{aligned}
$$

$$
\begin{aligned}
\left(\frac{\mathrm{d}^{2} \lambda_{3}}{\mathrm{dt}^{2}}\right)_{\mathrm{o}}= & {\left[\begin{array}{l}
3 \mathrm{~J}_{2}\left(\frac{\mathrm{a}_{\mathrm{e}}}{\mathrm{a}_{3}}\right)^{2}\left(1+\frac{7}{4} \mathrm{e}_{3}^{2}-7 \gamma_{3}^{2}\right) \\
+\mathrm{a}_{3}^{-4}\left(-\frac{15}{4} \mathrm{~J}_{4}+\frac{160}{11} \mathrm{~J}_{2}^{2}\right)
\end{array}\right] \frac{\mathrm{dn_{3 }}}{\mathrm{dt}} } \\
& -42 \mathrm{~J}_{2} \mathrm{n}_{3} \gamma_{3}\left(\frac{\mathrm{a}_{\mathrm{e}}}{\mathrm{a}_{3}}\right)^{2} \frac{\mathrm{d} \gamma_{3}}{\mathrm{dt}} \\
\left(\frac{\mathrm{d}^{2} \varphi}{\mathrm{dt}^{2}}\right)_{\mathrm{o}}= & \left(\frac{\mathrm{d}^{2} \lambda_{1}}{\mathrm{dt}^{2}}\right)_{\mathrm{o}}-2\left(\frac{\mathrm{d}^{2} \lambda_{3}}{\mathrm{dt}^{2}}\right)_{\mathrm{o}}+\frac{1}{2}\left\{\left(\frac{\mathrm{d}^{2} \Omega_{1}}{\mathrm{dt}^{2}}\right)_{\mathrm{o}}+\left(\frac{\mathrm{d}^{2} \Omega_{3}}{\mathrm{dt}^{2}}\right)_{\mathrm{o}}\right\}
\end{aligned}
$$

$$
\left(\frac{\mathrm{d}^{2} \sigma}{\mathrm{dt}^{2}}\right)_{\mathrm{o}}=\frac{1}{2}\left(\frac{\mathrm{d}^{2} \Omega_{1}}{\mathrm{dt}^{2}}\right)_{\mathrm{o}}-\frac{3}{2}\left(\frac{\mathrm{d}^{2} \Omega_{3}}{\mathrm{dt}^{2}}\right)_{\mathrm{o}}+\left(\frac{\mathrm{d}^{2} \varpi_{3}}{\mathrm{dt}^{2}}\right)_{\mathrm{o}}
$$

\begin{tabular}{|c|c|c|c|c|c|c|c|c|c|}
\hline & $m=\frac{M_{i, i=1-6}}{M}$ & $n(\mathrm{rad} / \mathrm{yr})$ & $i(\mathrm{deg})$ & $M_{S} / M$ & $a_{e}(\mathrm{~km})$ & $E$ & $\mathrm{~J}_{2}$ & $\mathrm{~J}_{4}$ & $\mathrm{~J}_{6}$ \\
\hline Mimas & $6.34 \times 10^{-8}$ & 2422.44 & 1.62 & - & - & 0.0194 & & & \\
\hline Enceladus & $0.15^{\times 10^{-6}}$ & & & & & & & & \\
\hline Tethys & $1.06 \times 10^{-6}$ & 1213.17 & 1.093 & - & - & 0.009 & & & \\
\hline Dione & $1.963 \times 10^{-6}$ & & & & & & & & \\
\hline Rhea & $4.32 \times 10^{-6}$ & - & - & - & - & - & & & \\
\hline Titan & $236.638 \times 10^{-6}$ & & & & & & & & \\
\hline Saturn & - & - & - & 3498.79 & 60330 & - & $\begin{array}{c}0.012 \\
98\end{array}$ & $\begin{array}{c}0.000 \\
915\end{array}$ & $\begin{array}{c}0.000 \\
095\end{array}$ \\
\hline
\end{tabular}

\begin{tabular}{|c|c|c|c|c|c|}
\hline $\mathrm{i}-\mathrm{j}$ & $\alpha_{\mathrm{ij}}$ & $\mathrm{A}\left(\alpha_{\mathrm{ij}}\right)$ & $\mathrm{B}\left(\alpha_{\mathrm{ij}}\right)$ & $C\left(\alpha_{i j}\right)$ & $\frac{d A\left(\alpha_{i j}\right)}{d \alpha_{i j}}$ \\
\hline $1-2$ & 0.78026 & 1.2473 & 1.3674 & -5.4695 & 1.0996 \\
\hline $1-3$ & 0.63064 & 1.1306 & 0.38952 & -1.5581 & 0.55451 \\
\hline $1-4$ & 0.49258 & 1.0706 & 0.15366 & -0.61463 & 0.33609 \\
\hline $1-5$ & 0.35283 & 1.0335 & 0.059940 & -0.23976 & 0.20479 \\
\hline $1-6$ & 0.15223 & 1.0059 & 0.0090810 & -0.036324 & 0.078148 \\
\hline $2-3$ & 0.80824 & 1.2807 & 1.8535 & -7.4138 & 1.2978 \\
\hline $3-4$ & 0.78108 & 1.2482 & 1.3790 & -5.5159 & 1.1047 \\
\hline $3-5$ & 0.55948 & 1.0960 & 0.23856 & -0.95425 & 0.42538 \\
\hline $3-6$ & 0.24140 & 1.0151 & 0.024460 & -0.097840 & 0.12912 \\
\hline
\end{tabular}

Table 1: Analytical Expressions of the Functions $f_{i}\left(\alpha_{13}\right)$ for the Arguments $\varphi, \psi+\varphi, \psi, \psi-\varphi, \chi$, with Their Value for $\alpha 13=0.6306386($ See $[11])$

\begin{tabular}{llll}
\hline Argument & $\mathrm{i}$ & $\mathrm{f}_{\mathrm{i}}(\alpha)$ & $\mathrm{f}_{\mathrm{i}}\left(\alpha_{13}\right)$ \\
\hline$\varphi$ & 0 & $-\alpha_{13} \mathrm{~b}_{3 / 2}^{3}\left(\alpha_{13}\right)$ & -1.65088068 \\
$\psi+\varphi$ & 1 & $3 \alpha_{13} \mathrm{~b}_{3 / 2}^{4}\left(\alpha_{13}\right)+\frac{1}{4}\left(\alpha_{13}\right)^{2} \frac{\mathrm{d}}{\mathrm{d} \alpha} \mathrm{b}_{3 / 2}^{4}\left(\alpha_{13}\right)$ & 5.23786953 \\
$\psi$ & 2 & $2 \alpha_{13} \mathrm{~b}_{3 / 2}^{2}\left(\alpha_{13}\right)+\frac{1}{2}\left(\alpha_{13}\right)^{2} \frac{\mathrm{d}}{\mathrm{d} \alpha} \mathrm{b}_{3 / 2}^{2}\left(\alpha_{13}\right)$ & 9.70821605 \\
$\psi-\varphi$ & 3 & $-\alpha_{13} \mathrm{~b}_{3 / 2}^{2}\left(\alpha_{13}\right)+\frac{1}{4}\left(\alpha_{13}\right)^{2} \frac{\mathrm{d}}{\mathrm{d} \alpha} \mathrm{b}_{3 / 2}^{2}\left(\alpha_{13}\right)$ & 0.22188903 \\
$\chi$ & 4 & $\frac{1}{2} \alpha_{13} \mathrm{~b}_{3 / 2}^{3}\left(\alpha_{13}\right)=-\mathrm{f}_{0}\left(\alpha_{13}\right)$ & 0.82544034 \\
\hline
\end{tabular}

Table 2 : Parameters of Mimas, Enceladus, Tethys, Dione, Rhea, Titan and Saturn $\mathrm{M}_{\mathrm{i}, \mathrm{i}=1-6}$, M and $\mathrm{M}_{\mathrm{S}}$ are the Masses of the Considered Satellite, Saturn and the Sun, Respectively

Table 3: Values of $\alpha_{i j}, A\left(\alpha_{i j}\right), B\left(\alpha_{i j}\right), C\left(\alpha_{i j}\right), \frac{d A\left(\alpha_{i j}\right)}{d \alpha_{i j}}$ for Every Pair $(i, j),(i<j)$ Involving Mimas, Enceladus, Tethys, Dione, Rhea and Titan. $\left(\alpha_{i j}\right)$ (See [3] and [4]) 


\section{Numerical integration and surface of sec-} tions

Our equations were integrated backwards in time. The initial conditions are taken from Vienne and Duriez [3,4] for J2000.
$\mathrm{i}_{1}=1.62^{\mathrm{O}}, \mathrm{i}_{3}=1.093^{\mathrm{O}}$ and $\mathrm{e}_{3}=0.0008$ are taken to be fixed. Here we have integrated it for $-62.8 \times 14000 \mathrm{yrs}$.

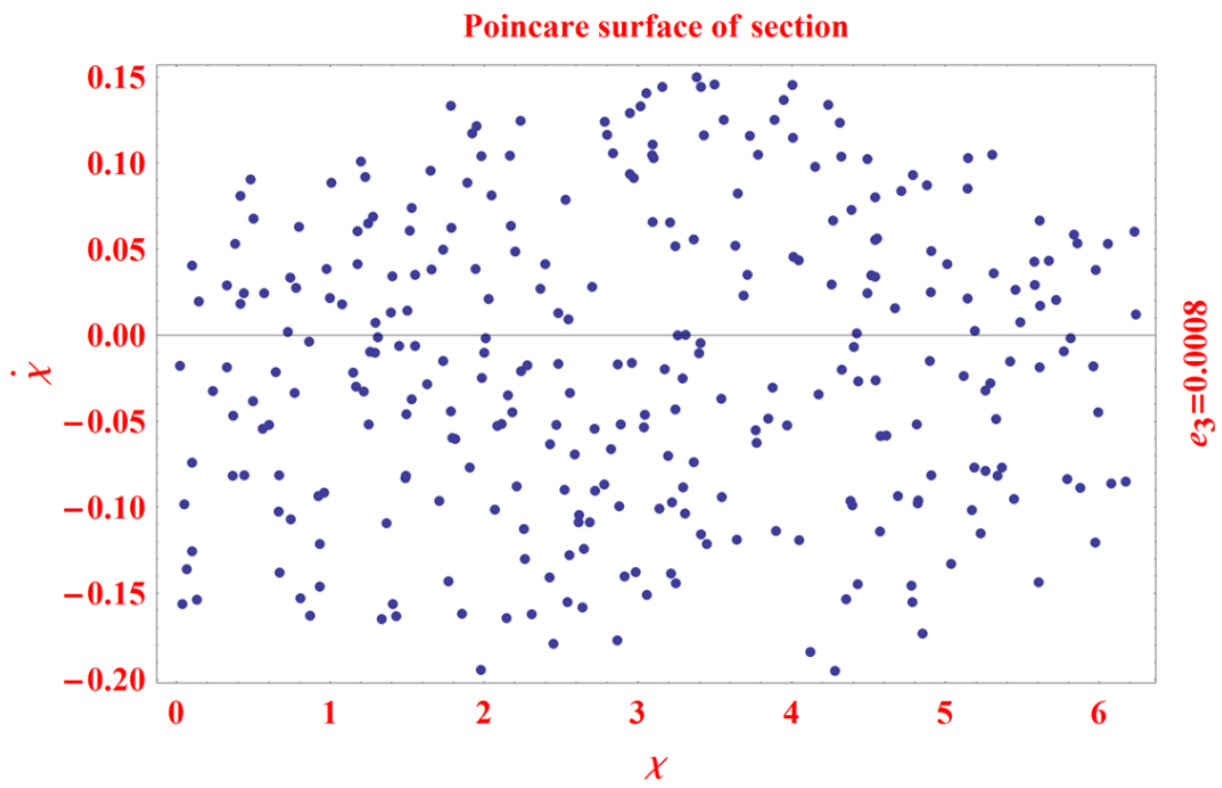

Fig. 4.1: Poincare Surface of Section at $i_{3}{ }^{2}, i_{1}{ }^{2} e_{3}, i_{1} i_{3} e_{3}$ and $i_{3}{ }^{2} e_{3}$ Resonances with Oblateness of Saturn. Secular Resonances of All Inner Satellites Have Been Considered at $\mathrm{i}_{1}=1.62^{\mathrm{O}}, \mathrm{i}_{3}=1.093^{\mathrm{O}}$ and $\mathrm{e}_{3}=0.0008$.

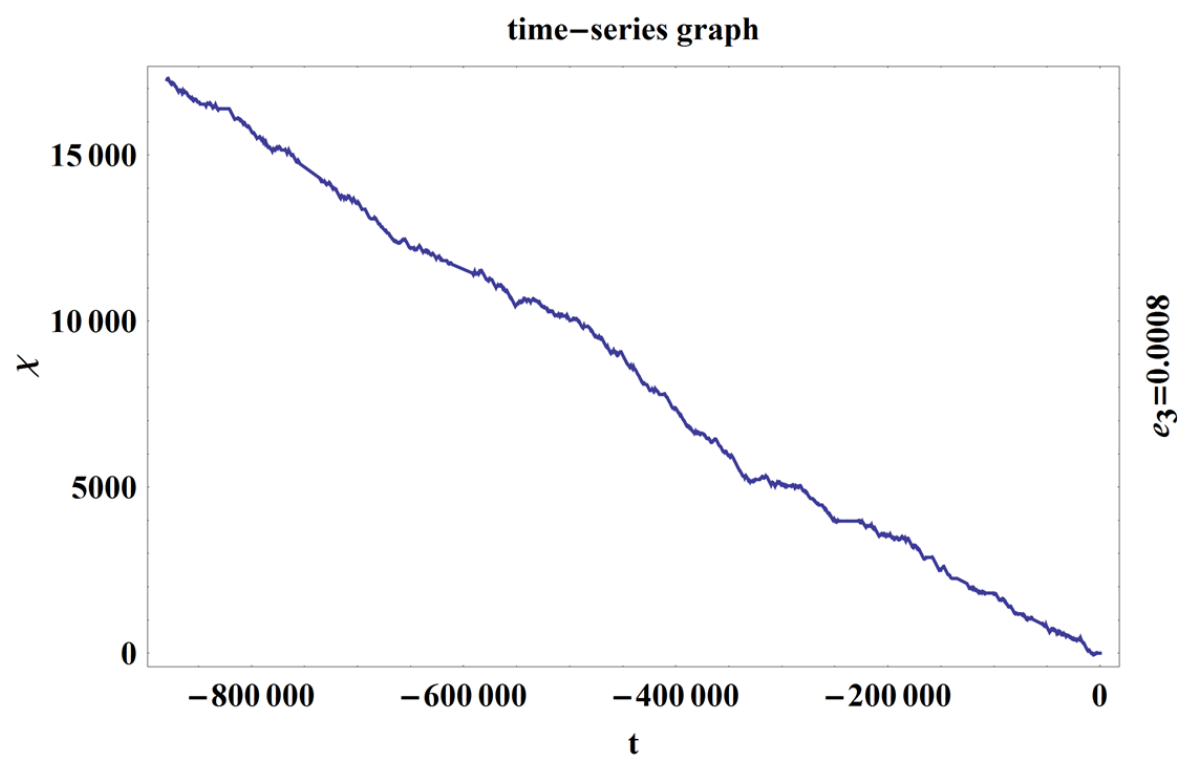

Fig. 4.2: Time-Series Graph at $\mathrm{i}_{3}{ }^{2}, \mathrm{i}_{1}{ }^{2} \mathrm{e}_{3}, \mathrm{i}_{1} \mathrm{i}_{3} \mathrm{e}_{3}$ and $\mathrm{i}_{3}{ }^{2} \mathrm{e}_{3}$ Resonances with Oblateness of Saturn. Secular Resonances of All Inner Satellites Have Been Considered at $\mathrm{i}_{1}=1.62^{\mathrm{o}}, \mathrm{i}_{3}=1.093^{\mathrm{o}}$ and $\mathrm{e}_{3}=0.0008$. 


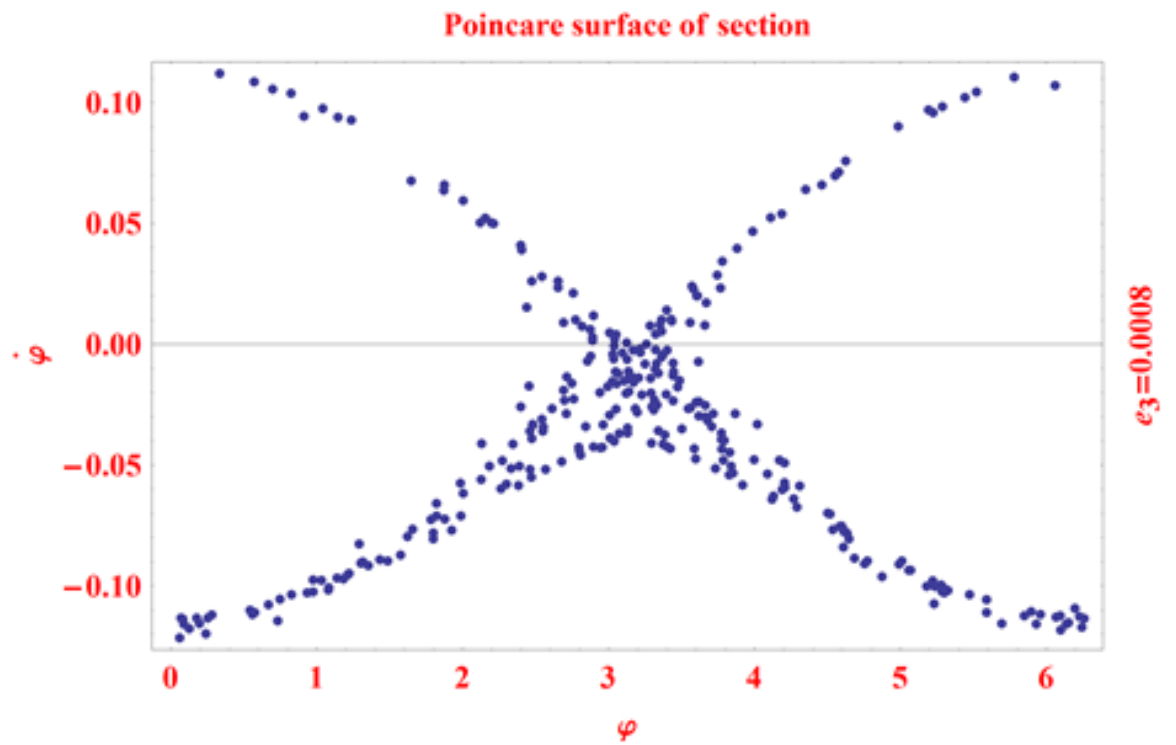

Fig. 4.3: Poincare Surface of Section at $\mathrm{i}_{1} \mathrm{i}_{3}, \mathrm{i}_{1}^{2} \mathrm{e}_{3}, \mathrm{i}_{1} \mathrm{i}_{3} \mathrm{e}_{3}$ and $\mathrm{i}_{3}{ }^{2} \mathrm{e}_{3}$ Resonances with Oblateness of Saturn. Secular Resonances of All Inner Satellites Have Been Considered at $\mathrm{i}_{1}=1.62^{\mathrm{O}}, \mathrm{i}_{3}=1.093^{\mathrm{O}}$ and $\mathrm{e}_{3}=0.0008$.

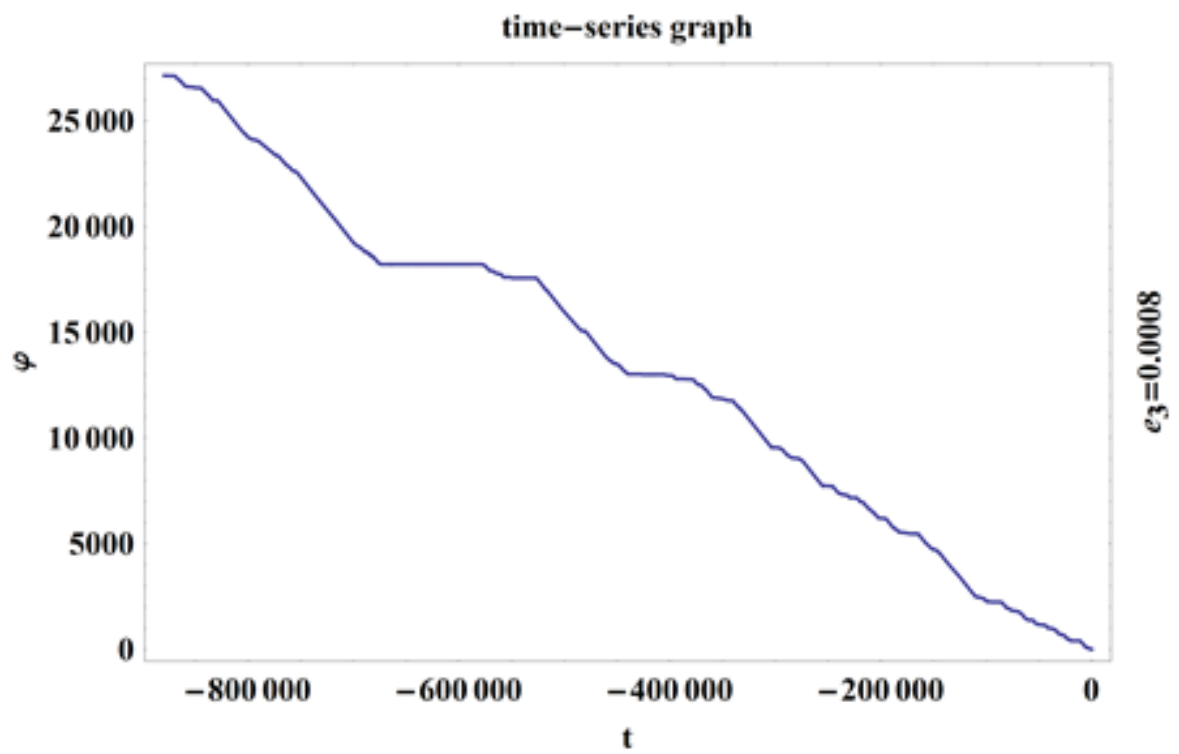

Fig. 4.4: Time-Series Graph at $\mathrm{i}_{1} \mathrm{i}_{3}, \mathrm{i}_{1}{ }^{2} \mathrm{e}_{3}, \mathrm{i}_{1} \mathrm{i}_{3} \mathrm{e}_{3}$ and $\mathrm{i}_{3}{ }^{2} \mathrm{e}_{3}$ Resonances with Oblateness of Saturn. Secular Resonances of All Inner Satellites Have Been Considered at $\mathrm{i}_{1}=1.62^{\mathrm{O}}, \mathrm{i}_{3}=1.093^{\mathrm{O}}$ and $\mathrm{e}_{3}=0.0008$.

\section{Discussion}

Vienne and Duriez [3] discovered the 200 year period in the mean longitude of Mimas. Champenois and Vienne $[5,6]$ have investigated the role of 200 year long period on the dynamics of Mimas-Tethys system when considered to be presently trapped in $i_{1} i_{3}$ resonance with probability of capture 0.76 at $2: 4$ commensurability. They found that the sources of this period are both the oblateness of Saturn and the interaction between its six inner satellites. They also realized that considering an eccentric orbit of Tethys upsets the earlier vision of dynamics of the Mimas-Tethys system. Here we have analyzed that the system was more chaotic if considered to be (at presently it is) locked in $i_{1} i_{3}$ resonance compared with $i_{3}{ }^{2}$ resonance, we considered the effect of
Saturns oblateness and the interaction of its six inner satellites, by the help of Poincare surface of section and timeseries graphs which confirms the earlier results too. Jha and Agrawal [5] also got the same result without considering the secular resonance of all inner satellite and Saturn's oblateness.

\section{References}

[1] Allan.R.R (1969). "Evolution of Mimas-Tethys commensurabilities", Astronomical Journal, 74, pp.997-506. https://doi.org/10.1086/110827.

[2] Sinclair.A.T. (1972). "On the origin of the commensurabilities amongst the satellites of Saturn", Mon. Not. R. Astrn. Soc., 160, pp.169-187. https://doi.org/10.1093/mnras/160.2.169.

[3] Vienne.A and Duriez.L (1992, a). "A general theory of Motion for The Eight Major satellites of Saturn", Astronomy and Astrophysics, 257, pp-331-352. 
[4] Vienne.A and Duriez.L (1992,b). "TASS1.6, Ephemerides of the major saturnian satellites", Astronomy and Astrophysics, 297, pp588-605.

[5] Champenois.S and Vienne.A (1999, a). "The Role of secondary resonances in the evolution of the Mimas-Tethys system", Icarus, 140(1), pp.106-121. https://doi.org/10.1006/icar.1999.6115.

[6] Champenois.S and Vienne.A (1999, b). "Chaos and Secondary resonances in the Mimas-Tethys system", Celestial Mechanics and Dynamical Astronomy, 74, pp.111-146. https://doi.org/10.1023/A:1008314007365.

[7] Jha.S and Agrawal.S.K (2014, a). "Chaotic Dynamics of MimasTethys System due to Secular Resonance of Enceladus", GJDSA, 4(1), pp. 1-11.

[8] Jha.S and Agrawal.S.K (2014, b). "Effect of Second and Third order resonances on Mimas- Tethys system", IJAMM, 3(1), 9-21.

[9] Jha.G.K and Jha.S (2015). "Comparative study of Secular Resonance Effect of Enceladus and Dione on Mimas- Tethys System", IJAMM, 4(1), pp. 27-31.

[10] Jha.G.K and Jha.S (2016, a). "Combined Effect of Oblateness of Saturn and Secular Resonance of Rhea on Mimas-Tethys System", M0 (Accepted).

[11] Jha.G.K and Jha.S (2016, b). "Role of Saturn's Oblateness and Secular Resonance of Various Combination of Inner Satellites with Titan on Mimas-Tethys System", IJST (Accepted). 